\title{
ON A SINGULAR MEASURE OF D. M. CONNOLLY AND J. H. WILLIAMSON
}

\author{
by P. M. LUDVIK
}

(Received 5th August 1974)

In (1) a measure $\lambda \in M(R)$ is constructed and shown to satisfy the following:

(i) $\lambda * \tilde{\lambda}$ is absolutely continuous, where $\tilde{\lambda}$ denotes the measure with $\tilde{\lambda}(E)=\overline{\lambda\left(E^{-1}\right)}$ for all Borel sets $E$,

(ii) $\lambda * \lambda=\lambda^{2}$ is singular,

(iii) $\lambda^{k}=\lambda^{k-1} * \lambda$ is absolutely continuous for $k \geqq 6$.

The purpose of this note is to show that (iii) can be sharpened to read "for $k \geqq 3$ ".

Let us now fix some notation and show how the measure $\lambda$ has been constructed. Denote by $G$ the complete direct product $\prod_{r=1}^{\infty} G_{r}$, where $G_{r}$ are finite cyclic groups $\simeq \mathbb{Z}\left(q_{r}\right)$. There is a natural continuous map from $G$ into $\mathbb{R}$ (or $T$ ) given by $g \rightarrow \sum_{r=1}^{\infty} g_{r} . d_{r}^{-1}$, where $d_{r}=q_{1} q_{2}, \ldots, q_{r}$. This map, $\phi$ say, gives rise to a map between $M(G)$ and $M(R)(M(T))$ by $\phi(\mu)[f]=\mu[f \circ \phi]$ for $f \in C_{0}(\mathbb{R}), \mu \in M(G)$.

In (1) the authors have chosen $q_{r}=a_{r}^{2}+a_{r}+1, a_{r}$ is a power of a prime $p_{r}$, and Singer's theorem shows the existence of sets $X_{r} \subset G_{r}$ such that

(i) card $X_{r}=a_{r}+1$,

(ii) $X_{r}-X_{r}=\left\{x_{1}-x_{2} \mid x_{1} \in X_{r}, x_{2} \in X_{r}\right\}=G_{r}$,

(iii) $\sum_{r=1}^{\infty} a_{r}^{-1}<\infty$.

If we now take $\mu_{r}$ to be the uniform probability measure supported on $X_{r}$, i.e.

$$
\begin{array}{lll}
\mu_{r}\{x\}=\left(a_{r}+1\right)^{-1} & \text { for } & x \in X_{r} \\
\mu_{r}\{x\}=0 & & x \notin X_{r}
\end{array}
$$

and take $\mu \in M(G)$ to be $\mu=\bigotimes_{r=1}^{\infty} \mu_{r}$, the unique product measure corresponding to $\left\{\mu_{r}\right\}_{r=1}^{\infty}$, then $\lambda$ is just the image $\phi(\mu)$ of $\mu$.

G. Brown and W. Moran have established the following remarkable result (2, lemma 5, p. 12): 
Let $\mu_{r}=m_{r}+\rho_{r}, m_{r}$ is the normalised Haar measure on $G_{r}, \mu_{r}$ a probability measure on $G_{r}$. Let $\mu=\bigotimes_{r=1}^{\infty} \mu_{r}$ and let $\phi(\mu)=v \in M(T)$. Write

$$
\alpha_{r}(k)^{2}=\int_{G_{r}}\left|\frac{d \rho_{r}^{k}}{d m_{r}}\right|^{2} d m_{r}=\int_{G_{r}}\left|\left(\rho_{r}^{k}\right)^{\wedge}(\gamma)\right|^{2} d \gamma=\sum_{\gamma \in G_{r}}\left|\left(\rho_{r}^{k}\right)^{\wedge}(\gamma)\right|^{2} .
$$

Then, if $\sum_{r=1}^{\infty} \alpha_{r}(k)^{2}<\infty, v^{k}$ is absolutely continuous, i.e. $v^{k} \in L^{1}(T)$.

Let us now come back to the results of (1). Then $\lambda=\phi(\mu)$, where $\mu=\otimes \mu_{r}$ and $\mu_{r}$ are as specified above. Despite the fact that we do not know much about the sets $X_{r}$ we can give a very precise description of $\mu_{r} * \tilde{\mu}_{r}$. It is trivial to see that

$$
\mu_{r} * \tilde{\mu}_{r}=\frac{1}{\left(a_{r}+1\right)^{2}}\left\{\left(a_{r}+1\right) \delta_{0}+\sum_{g \neq 0} \delta_{g}\right\}
$$

where $\delta_{g}$ is the unit mass of point $g$. So

$$
\begin{aligned}
\mu_{r} * \tilde{\mu}_{r} & =\frac{1}{\left(a_{r}+1\right)^{2}}\left\{a_{r} \delta_{0}+\sum_{g \in G_{r}} \delta_{g}\right\} \\
& =\frac{1}{\left(a_{r}+1\right)^{2}}\left\{a_{r} \delta_{0}+\left(a_{r}^{2}+a_{r}+1\right) m_{r}\right\} \\
& =m_{r}+\left\{\frac{a_{r}}{\left(a_{r}+1\right)^{2}} \delta_{0}-\frac{a_{r}}{\left(a_{r}+1\right)^{2}} m_{r}\right\} \\
& =m_{r}+\rho_{r} * \tilde{\rho}_{r}
\end{aligned}
$$

when $\mu_{r}=m_{r}+\rho_{r}$.

Thus

and so

$$
\rho_{r} * \tilde{\rho}_{r}=\frac{a_{r}}{\left(a_{r}+1\right)^{2}} \delta_{0}-\frac{a_{r}}{\left(a_{r}+1\right)^{2}} m_{r}
$$

$$
\begin{array}{rlrl}
\left(\rho_{\mathrm{r}} * \tilde{\rho}_{\mathrm{r}}\right)^{\wedge}(\gamma)=\left|\hat{\rho}_{\mathrm{r}}(\gamma)\right|^{2} & =\frac{a_{\mathrm{r}}}{\left(a_{\mathrm{r}}+1\right)^{2}} & & \text { if } \gamma \neq 0 \\
& =0 & \text { if } \gamma=0 .
\end{array}
$$

Hence the criterion of Brown and Moran takes the form

$$
\alpha_{r}(k)^{2}=\sum_{\gamma \in G_{r}}\left|\left(\rho_{r}^{k}\right)^{\wedge}(\gamma)\right|^{2}=\sum_{\gamma \in G_{r}}\left(\left|\hat{\rho}_{r}(\gamma)\right|^{2}\right)^{k}=\left(a_{r}^{2}+a_{r}\right) \cdot a_{r}^{k} /\left(a_{r}+1\right)^{2 k}
$$

since card $\hat{G}_{r}=\operatorname{card} G_{r}$. So $\alpha_{r}(k)^{2} \sim a_{r}^{2-k}$ and since by (iii) $\sum_{r=1}^{\infty} a_{r}^{-1}<\infty$ we have that $\sum_{r=1}^{\infty} \alpha_{r}(k)^{2}<\infty$ for $k \geqq 3$, which implies that $\lambda^{k}$ is absolutely continuous for $k \geqq 3$. 


\section{REFERENCES}

(1) D. M. Connolly and J. H. Wirliamson, An application of a theorem of Singer, Proc. Edinburgh Math. Soc. 19 (1974), 119-123.

(2) G. Brown and W. Moran, Coin tossing and powers of singular measures, Proc. Cambridge Philos. Soc., to appear.

\section{UNIVERSITY OF YORK}

